\title{
Demonstration of a geode by magnetic resonance imaging: a new light on the cause of juxta-articular bone cysts in rheumatoid arthritis
}

\author{
E A Moore, R K Jacoby, R E Ellis, M E Fry, S Pittard, W Vennart
}

\begin{abstract}
The magnetic resonance imaging (MRI) features of a rheumatoid arthritic geode are presented. Development of such a cyst from before $x$ ray diagnosis to its coalescence with the wrist joint is described. The evidence suggests that these juxta-articular cysts are not merely an intrusion of the synovial cavity into the bone marrow but start as isolated structures beneath the subchondral bone.
\end{abstract}

A number of authors have reported arthritic changes in bones and joints using magnetic resonance imaging (MRI) techniques. ${ }^{12}$ The exciting potential of MRI is its ability to disclose both bone surface and intra-articular material, such as cartilage, synovial membrane, and synovial fluid. As part of an extended MRI study to characterise the disease both qualitatively and quantitatively, through spin-lattice $\left(T_{1}\right)$ and spin-spin $\left(T_{2}\right)$ relaxation time measurements, we describe the findings for one patient with rheumatoid arthritis whose MRI scans showed a cyst or geode in the right wrist. In addition, we have made quantitative measurements of $T_{1}$ from the geode and surrounding tissues over a period of two years. The left wrist developed a similar cyst during the study and was also scanned with MRI. The investigation of this patient, we believe, sheds some light on the development of geodes in general.

\section{MRI system}

The MRI system was built in-house ${ }^{34}$ and is based on a $180 \mathrm{~mm}$ bore $0.5 \mathrm{~T}$ superconducting magnet, which enables images of the hand and wrist to be generated. Patients are able to sit comfortably with their hands in the magnet at the centre of the field. It is necessary to enclose the patient in an electrically screened room to eliminate extraneous radiofrequency signals from the system. All the images generated are from sections of the wrist about $3.5 \mathrm{~mm}$ thick. The images displayed here are obtained using saturation recovery sequences $(T R=800 \mathrm{~ms}$ ). Quantitative measurements were obtained by calculating $T_{1}$ values of specific areas using both saturation and inversion recovery sequences. ${ }^{5}$

\section{Case report}

A 41 year old white woman with a six year history of classical seropositive rheumatoid arthritis, ${ }^{6}$ responding to penicillamine $375 \mathrm{mg}$ a day, had pain and swelling in the wrists. At the onset of her arthritis she had been treated with azathioprine $50 \mathrm{mg}$ twice daily and corticotrophin (ACTH) 20 units daily. The ACTH was reduced to 20 units on alternate days after six months and ultimately withdrawn after four years. The azathioprine was withdrawn after three years and treatment with penicillamine started because of reactivation of synovitis in her wrists and hands. The symptoms and signs regressed until she relapsed again with painful wrists in March 1987.

Physical examination at that time showed synovial thickening of both wrists with pain and limitation of movement. The right wrist flexed to $20^{\circ}$, the left to $55^{\circ}$. Extension was equal in both at $50^{\circ}$. Radial and ulnar deviation in the right wrist was $20^{\circ}$ and in the left wrist it was $30^{\circ}$.

Investigations at that time showed haemoglobin $132 \mathrm{~g} / \mathrm{l}$, white blood cell count $9 \cdot 1 \times 10^{9} / \mathrm{l}$, erythrocyte sedimentation rate (ESR) $44 \mathrm{~mm} / \mathrm{h}$. Radiographs of the wrists showed loss of cartilage between the radius and carpus. In the right wrist a geode was present in the radius opposite the proximal end of the scaphoid (fig 1A). The scaphoid showed erosive changes, but these were not at its proximal pole. Magnetic resonance images clearly showed degradation of cartilage within the wrist (fig 1B) and a cavity within the distal end of the radius was clearly visible. The left wrist had no such cavity (fig 1C). The cavity in the right radius seemed to be separate from the joint space and was defined by an outline of cortical bone (black area on the magnetic resonance image), which was confirmed by the radiograph.

The amount of cortical bone around the geode varied with the level of the section. At the centre of the geode the intense signal characteristic of static fluid was seen with a $T_{1}$ value long compared with bone marrow. As the rim was approached a reduction in the signal intensity occurred, which equated with cortical bone. The contour of the cavity showed it to be roughly spherical. The mean $T_{1}$ of the geode contents (table) was found to be 417 (SD 63)

\begin{tabular}{lll}
$\begin{array}{l}T, \text { values of tissues. } \\
\text { (ms) }\end{array}$ & Values are means $(S D)$ in milliseconds \\
\hline Tissue & March 1987 & March 1989 \\
\hline $\begin{array}{l}\text { Right wrist } \\
\text { Marrow } \\
\text { Joint space }\end{array}$ & $145(20)$ & $186(2)$ \\
$\begin{array}{l}\text { Geode } \\
\text { Left wrist }\end{array}$ & $417(60)$ & $367(24)$ \\
Marrow & & $441(19)$ \\
Joint space & $147(35)$ & $155(1)$ \\
Geode & $365(108)$ & $332(10)$ \\
\hline
\end{tabular}

Normal values: bone marrow $170(20) \mathrm{ms}$; joint space $240(51)$ ms.
EX2 4UE.

Accepted for publication 12 December 1989

Medical Physics Group,
Department of Physics,
University of Exeter
E A Moore
R E Ellis
M E Fry
S Pittard
W Vennart
Royal Devon and Exeter
Hospital and
Torbay Hospital
R K Jacoby
Correspondence to:
Dr R K Jacoby,
Princess Elizabeth
Orthopaedic Hospital,
Exerer,
EX2 4UE.
Accepted for publication
12 December 1989


Figure 1 March 1987 scans. (A) Radiograph of the right wrist, showing a geode in the radius (arrow) (curved arrow) and loss of joint space (small arrows). (C) Coronal section of left wrist; loss of joint space is shown (arrows)

$\mathrm{Pr}=$ proximal $; \mathrm{D}=$ distal $L=$ lateral $; M=$ medial . (B) Coronal section of right wrist, showing the geode

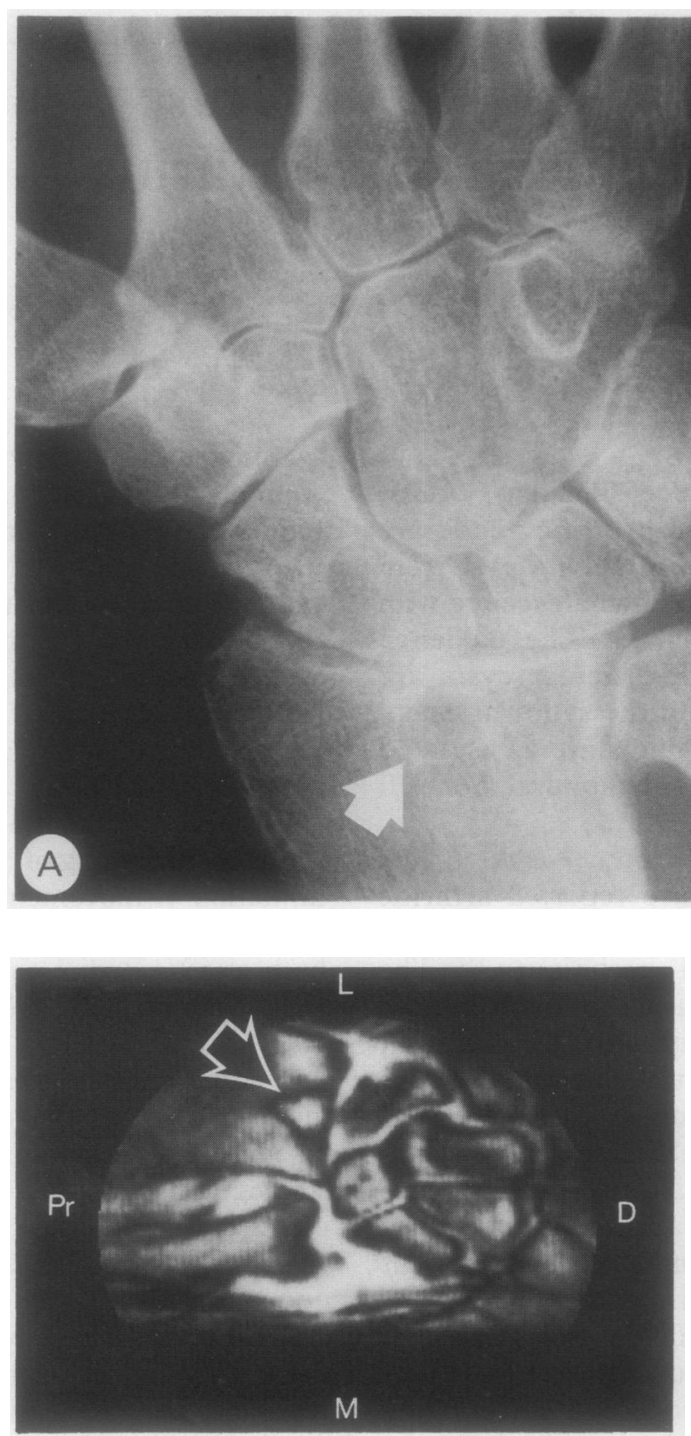

Figure 2 March 1988 Coronal section of right wrist, showing the geode is enlarged (arrow) but separated from the join space by cortical bone. Pr $=$ proximal; $D=$ distal; $L=$ lateral $; M=$ medial .

Figure 3 March 1989 Right wrist, $(A)$ coronal section and $(B)$ sagittal section, showing the geode is further enlarged and is continuous with the joint space (arrows).

Pr=proximal; $D=$ distal; $L=$ lateral $; M=$ medial $A=$ anterior $; P o=$ posterior.
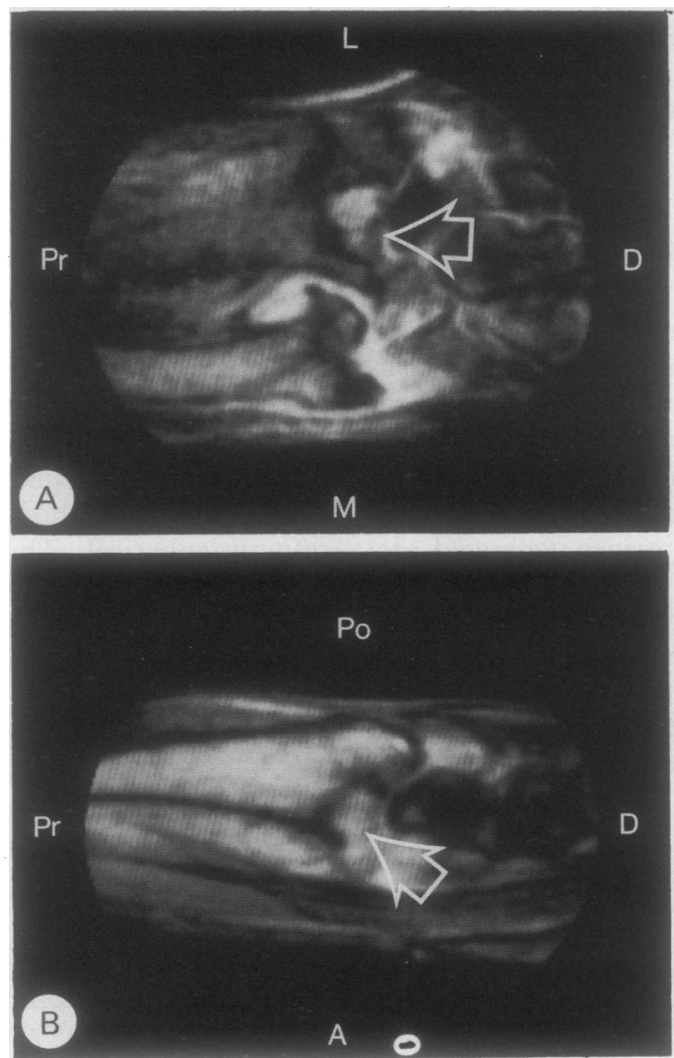

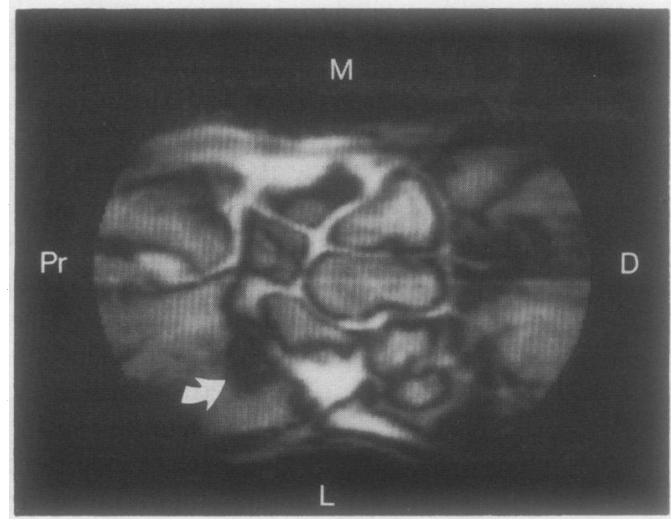

Figure 4 March 1988. Coronal section of left wrist. A small geode is apparent in the radius (arrow) and is not connected with the joint space. $P r=$ proximal; $D=$ distal;

$\boldsymbol{L}=$ lateral $; \boldsymbol{M}=$ medial .
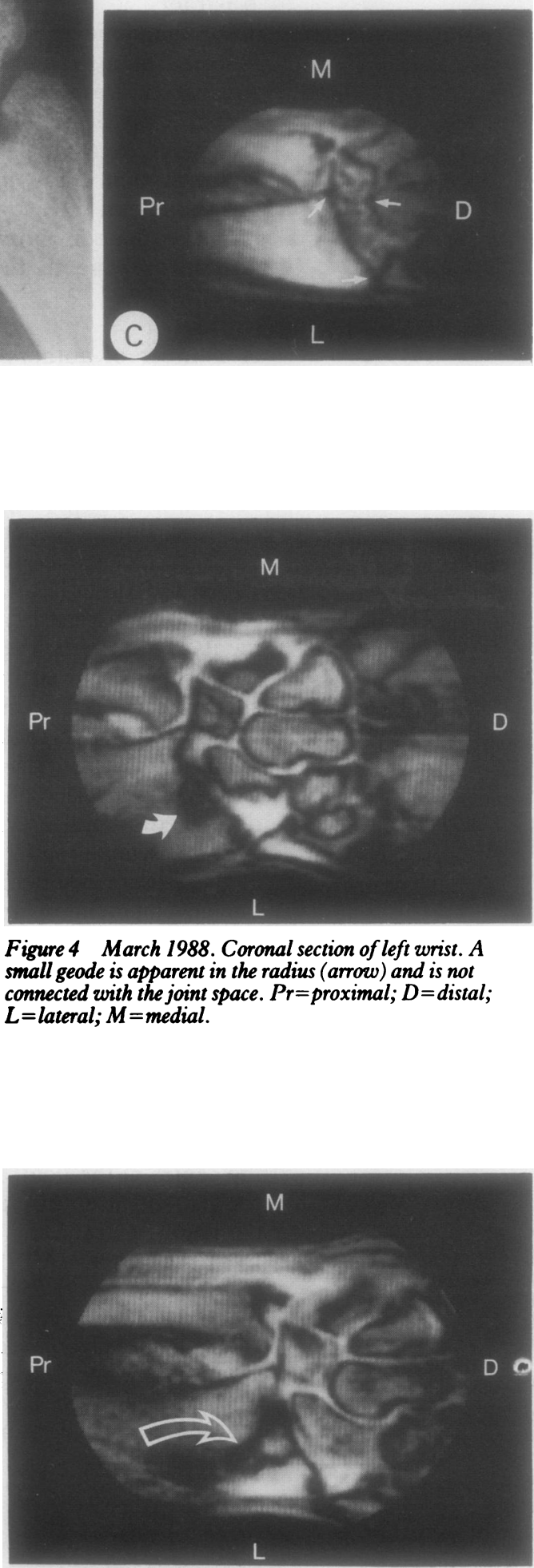

Figure 5 March 1989. Coronal section of left wrist, showing the geode is enlarged (arrow). $T$, values indicate it is continuous with the joint space, though this is not shown on this section. 
milliseconds (ms) while that of surrounding bone marrow was 145 (20) ms; bone marrow $T_{1}$ values in the range of 150 to $190 \mathrm{~ms}$ were obtained from a group of normal volunteers. After 12 months the geode had increased in size (fig 2), but $T_{1}$ measurements were not made on this occasion. After a further 12 months the geode had developed from an isolated cyst, both in terms of the image and $T_{1}$ measurements, to one connected with the joint space (figs $3 \mathrm{~A}$ and 3B). The $T_{1}$ of the bone marrow had increased slightly to $186(2) \mathrm{ms}$, while the $T_{1}$ of the cyst, 441 (19) ms, had almost equalised with that of the joint space (367 (24) ms).

In the left wrist at the time of the first scan the bone marrow had the same $T_{1}$ as the right wrist-147 (35) ms, though the joint space was slightly higher at 365 (108) ms (normal range 200 to $300 \mathrm{~ms}$ ). The second scans showed a small geode in the left wrist, having a similar outline of cortical bone and a fluid filled centre (fig 4). At the final scans the size of this geode had also increased (fig 5) and the $T_{1}$ values of the geode and joint space were about the same (324 (14) ms and 332 (10) ms respectively), indicating that the geode had become continuous with the joint space.

\section{Discussion}

Synovitis is associated with raised intra-articular pressure both at rest and with loading across the joint. ${ }^{7}$ Synovial cysts commonly form in response to the pressures developed within the rheumatoid joint and have been recognised for more than a century. ${ }^{8}$ Indeed it has been suggested that synovial cysts protect the joints and bone ends. ${ }^{9}$ In addition, if the articular cartilage and epiphysis are sufficiently damaged by the intraarticular hypertension, geodes or bone cysts may develop. ${ }^{10}$ The vexed question as to whether the cysts can develop before they communicate with the joint space is not yet answered. The data presented here, however, do shed some light on this problem. The magnetic resonance images of the right wrist show that the geode starts as an isolated area with a high $T_{1}$ compared with that of the surrounding bone marrow. The $T_{1}$ of the joint space is initially higher than normal, consistent with excess intra-articular fluid, and this increases further as the geode opens out into the joint. It should also be noted that the $T_{1}$ value of bone marrow in both wrists increases over the period of study. The $T_{1}$ value of the left wrist is not as high as that of the right but this might be due to developing osteoblastic activity resulting from the uneven stress patterns in trabecular bone, which may lead to further development of the geode as in the right wrist.

It is also of interest that the magnetic resonance image was able to detect cyst formation in the left wrist before changes were seen on the radiograph. The magnetic resonance image showed an abnormal area and the $T_{1}$ value was higher than that of the surrounding bone marrow. The $3.5 \mathrm{~mm}$ slices through the geodes failed to show a connection with the joint space in the early stages of development. If the disease within the joint has any bearing on the development of geodes we would suggest that the nutrition of the epiphysial marrow is compromised in some way. The $T_{1}$ measurements confirm a biochemical alteration within the marrow before the bony cyst develops. If this is not the correct scenario the term 'geode' would be inappropriate and should be replaced with the more mundane term 'joint sinus'. The final stage of pathological development is indeed a joint sinus, which fills up with joint debris and synovial fluid, as described by Bywaters. ${ }^{11}$ The authors would like to thank Torbay Health Authority, South
West Regional Health Authority, and the Science and Engineering Research Council for financial support.
Rest Regional Health Authority, and the

1 Keonig H, Lucas D, Heissner R. The wrist: a preliminary report on high resolution MR imaging. Radiology 1986; 160: 463-7.

2 Weiss K L, Beltran J, Lubbers L M. High field MR surface coil imaging of the hand and wrist. Part II. Pathological coil imaging of the hand and wrist. Part II. Pathological correlations

3 Pittard S, Ellis R E, Jacoby R K, Vennart W. Nuclear magnetic resonance imaging using a microcomputer. Med Biol Eng Comput 1988; 26: 221-4.

4 Pittard S, Fry M E, Ellis R E, Moore E A, Vennart W. A low cost magnetic resonance imaging system. Foumal of Physics E: Scientific Instruments 1989; 22: 574-82.

5 Crawley A O, Henkelmann R M. A comparison of one-shot and recovery methods in $T_{1}$ imaging. Magnetic Resonance and Medicine 1988; 7: 23-34.

6 Ropes M W, Bennett G A, Cobb S, Jacox R, Jessar R A. 1958 revision of diagnostic criteria for rheumatoid arthritis. Bull Rheum Dis 1958; 9: 175-6.

7 Jayson M I V, Dixon A StJ. Intra-articular pressure in rheumatoid arthritis of the knee. III. Pressure changes during joint use. Ann Rheum Dis 1970; 29: 401-8.

8 Baker $W M$. The formation of abnormal synovial cysts in connection with the joints. St Bartholomew's Hospital Reports 1855; 21: 177-90.

9 Genovese G R, Jayson M I V, Dixon A StJ. Protective value of synovial cysts in rheumatoid knees. Ann Rheum Dis 1972; 31: 179-82.

10 Jayson M I V, Rubenstein D, Dixon A StJ. Intra-articular pressure and rheumatoid geodes (bone cysts). Ann Rheum Dis 1970; 29: 496-502.

11 Bywaters E G L. Radiological aspects of rheumatoid arthritis. The hand. Amsterdam: Excerpta Medica, 1964: 43. (International congress series, No 61.) 